\title{
Innovations in the field of medicine in the era of informatics
}

\author{
Innovaciones de la medicina en la era de la informática
}

Julio Sotelo*

Comité de Ética y Transparencia en la Relación Médico-Industria, Mexico City, Mexico

In the last 100 years, the humanity has witnessed great progresses in terms of anesthetic and surgical procedures, use of antibiotics, sedatives, antidepressants, vaccines, hormones, imaging studies, among countless scientific advances, which have favorably transformed the ancestral task of coming up against disease and suffering. The result has been dramatic: human being's life expectancy is more than double than one century ago, approaching an average of 80 years in most countries with a reasonable implementation of medical services.

The endless sequence of innovations has produced deep changes in the area of health and, consequently, in patient expectations regarding medical interventions, which currently are mostly directed to the prevention or transformation of the course of ancestral health conditions. As it would be too long to even outlining them, I will restrict myself to indicating that, during the $20^{\text {th }}$ century, that which probably will be called the "golden age of medicine" was consolidated.

Along with medical advances, in recent decades, technological advances have transformed the entire society in an unexpected way. Electricity, aviation, automobiles, radio, telephony, television and cinematography are examples of inventions that have radically modified the human condition. In conjunction with innovations in medicine, they generated a new era of prosperity. Although they have been accompanied by unprecedented scenarios, not without risks and unfavorable possibilities, their benefits and structural novelties have become part of daily life. Just to mention one example, aviation has brought along drastic sociological changes that are here to stay, and its transforming effect is now immovable for the entire society, which takes advantage of its countless benefits and tries to counteract its occasional unfavorable effects or harmful uses (such as its employment in warfare). We have called all this transformations "progress", a myriad of events that we have adopted and that have modified our reality.

At the dawn of the third decade of this millennium, we are contemplating, with amazement and enthusiasm, the most revolutionary technological advent in recent years, perhaps the most relevant of this century: the uncontainable implementation of information technologies, which are transforming the entire society and its structures. As it has always been the case with large-scale technological advances, medicine will be drastically modified by this technological revolution, which is so comprehensive that it will possibly justify the denomination "information technology age"; if so, it will be a turning point in the field of medicine.

With no doubt, the incorporation of information technology will change everything the doctor does for society and will influence his ancestral profession, directing it through paths that are unprecedented and were unimaginable one decade or two ago. Computing, cell phones and the internet have become part of society's daily life, and their transforming effect spreads to medical practice; it is just the beginning, but we can anticipate that it will follow an innovative, deep and, in many ways, unpredictable path. Medical science and ethics will be in charge of favorably incorporating information technology and neutralizing or limiting its risks; thus have they done for the last 100 years with many other technologies, which now 
are essential. The same seems to be the case with information technologies, which, without realizing it, are transforming medical practice to levels that were unexpected until recently. I will only refer, in a brief and limited way, some repercussions in medical science.

The cell phone has reinforced instant communication between doctors and patients, which was not possible with landline telephones. Now, the doctor can be located anytime and without obstacles by the patient; the benefits and potential overuse are obvious, but transformative in the doctor-patient relationship. The new mobile phone not only provides telephonic service, but also real-time photography, cinematography, information technology, computing, internet and video. In this device we can read, consult an encyclopedia or a dictionary or search for comprehensive scientific information; it is even a source of entertainment and trivial knowledge, as well as a connection to "social networks", which are democratic and individualized tools that have taken root in society and that are not without sharp edges.

These "benefits" integrated into mobile telephony are the cradle of modifying innovations the sole outline of which would lead to extended texts, but whose effects will be radical and, for now, unpredictable in their angles. We all know and anticipate that the information technology revolution is an irreversible process, the effects of which will also affect medicine, doctors and patients.

The advent and popularization of the internet have allowed obtaining complete, comprehensive, instantaneous scientific information and at the moment it is generated; this was not feasible a few years ago, not even for health professionals who practiced in the most modern medical facilities. Currently, medical scientific information on any subject can be accessed from any computer, even from any mobile phone. However, this information is accessible to health professionals just the same as to anyone who wishes to consult it. A novelty with enormous benefits for the practice and knowledge of medical progress, which is accompanied by multiple conjectures about the use of information, a circumstance that is also irreversible. Medical knowledge, particularly that related to specialties, until recently an exclusive domain of medical professionals, will have to adjust to liberal access to scientific information; the dilemma will be how to separate valuable information from trivial or redundant or inadequate or frankly risky data.

A rooted practice that is considered essential in professional training and updating is regular celebration of congresses, medical meetings and academic sessions, which have also been transformed by information sciences. Conferences and sessions with experts can now be integrated and completed by accessing the internet. The incorporation of this tool of information technology excellence into the training of medical science students -which should consider humanistic, ethical, cultural and optimal psychological development precepts for future professionals- is current subject of analysis and classical teaching methods modifying algorithms. Possible risks and deviations are, again, countless; the most conspicuous are academic isolation and "self-teaching" temptation in a complex discipline with multiple humanistic angles, where the acquisition and interpretation of data, although essential, constitute only one part of comprehensive medical practice.

In the coming decades, it will be possible to recognize how to take full advantage of information sciences and moderate their risks in scientific research and in the treatment and prevention of diseases and, moreover, to the benefit of society. 